\title{
Comparative Analyses of the Transcriptome and Proteome of Comte de Paris and Smooth Cayenne to Improve the Understanding of Ethephon-Induced Floral Transition in Pineapple
}

\author{
Chuan-He Liua,b,c Yan Liü, Xue-Hua Shao ${ }^{a, b, c}$ Duo Laia,b,c \\ anstitute of Fruit Tree Research, Guangdong Academy of Agricultural Sciences, Guangzhou, 'bey \\ Laboratory of South Subtropical Fruit Biology and Genetic Resource Utilization Ministry of Agriculture, \\ Guangzhou, 'Guangdong Province Key Laboratory of Tropical and Subtropical Fruit Tree Research, \\ Guangzhou, China
}

\section{Key Words}

Pineapple - Comte de Paris - Smooth Cayenne - Transcriptome - Proteome - Ethephoninduced floral transition

\begin{abstract}
Background/Aims: Ethylene is usually used to induce floral transition in pineapple. However, its successful induction in plants categorized as Cayenne is difficult or completely ineffective, and information concerned is limited. The present study was undertaken to investigate the molecular mechanisms underlying this obstacle. Methods: Transcriptome and proteome comparative analyses were performed to explore the important regulation and pathway variations after ethephon induction in the induction-easy 'Comte de Paris' (CP) and inductionhard 'Smooth Cayenne' (SC) cultivars via RNA-seq (RNA-sequencing) and iTRAQ (isobaric tags for relative and absolute quantification). Results: CP and SC exhibited basic differences at the transcriptomic and proteomic levels before ethephon treatment, including the expression of genes and proteins related to ethylene signal transduction. After ethephon induction, the expression of genes and proteins involved in plant ethylene signal transduction and carbohydrate metabolism responded more strongly in CP than in SC. The expression of the floral meristem identity (FMI) genes $A G, T F L$ and $F T$ exhibited greater changes in $C P$, and more transcription factors responded in SC. Gene Ontology (GO) and Kyoto Encyclopedia of Genes and Genomes (KEGG) enrichment analyses revealed that many differentially expressed genes (DEGs) in CP were annotated to terms and pathways involved in photoperiodism and shared components involved in carbohydrate metabolism and plant hormone signal
\end{abstract}

\begin{tabular}{ll}
\hline Chuan-He Liu & Institute of Fruit Tree Research, Guangdong Academy of Agricultural Sciences \\
& 510640, Guangzhou (China) \\
E-Mail founderlch@126.com
\end{tabular}


Liu et al.: Comparative Transcriptome and Proteome Analyses of Ethephon-Induced

Pineapple Floral Transition

transduction. Conclusion: These findings contribute to the understanding of the molecular mechanism underlying the variation between $\mathrm{CP}$ and SC in response to ethephon-mediated floral induction.

(c) 2018 The Author(s)

Published by S. Karger AG, Basel

\section{Introduction}

Pineapple [Ananas comosus (L.) Merr.], which is indigenous to Brazil, Argentina and Paraguay but has been introduced worldwide, is the leading edible member of the Bromeliaceae family and is one of the most important fruit crops in tropical and subtropical regions [1-2]. Pineapple fruit are economically important and highly valued because of their attractive sensorial and nutritional characteristics [3] and because they are a good source of antioxidants [4-6].

The transition from vegetative growth to flowering is important, as this is the first step of sexual reproduction and fruit set in agricultural and horticultural plants [7-8]. Pineapple reproductive development is induced by shortened day lengths and cool night temperatures and is mediated by a burst in ethylene production or increased ethylene sensitivity in the shoot apical meristem [9-12]. Artificial forcing of pineapple flowering is a well-established commercial practice that aims to synchronize pineapple flowering with ethylene or ethylenereleasing chemicals such as ethephon [(2-chloroethyl) phosphonic acid] as well as acetylenereleasing calcium carbide $\left(\mathrm{CaC}_{2}\right)$ [12-14].

At 48 hours after ethephon application, pineapple shoot apices start to show differentiation signs such as an incipient vacuolization of the cells, and that, at 72 hours, primordial leaves started to separate from each other; at 72 hours, small, densely colored cells at the meristematic dome periphery, which forms the so-called tunic-corpus structure, could be observed [12]. At eight days, the plants treated with ethephon showed signs of inflorescence development, in which the phyllome stopped developing; however, the apex widened, and the inflorescence primordium formed. Twelve days later, sepals, petals, pistils and stamens differentiated successively in the first-layer florets [15].

Apart from morphological concerns, leaf basal-white tissue was found to produce higher volumes of ethylene, abscisic acid (ABA) and 2-isopentyl adenine (2-iP) and reduce the contents of gibberellic acid $\left(\mathrm{GA}_{3}\right)$, indole-3-acetic acid (IAA) and zeatin (ZT) in the long term, which led to the transition from vegetative growth to inflorescence initiation [11-12, 15]. In addition, ethephon application caused an increase in the levels of proteins in buds treated after 60 hours, and of all the carbohydrates examined in that process, sucrose exhibited the greatest involvement [16].

Ethylene-related genes, including ethylene receptor genes (ETRs) and ethylene response factor genes ( $E R F \mathrm{~s}$ ), have been cloned from pineapple and have been found to be upregulated in response to ethephon stimulation in pineapple [12, 17-18]. Aminocyclopropane carboxylic acid (ACC) synthase (ACS) and ACC oxidase (ACO) genes have been shown to be key regulatory genes in the biosynthesis of ethylene [19-23]. AcACS2 and ACACO1 are upregulated by ethephon application and are thought to be among the key contributors of the triggering of flowering [12, 23-25].

Following floral initiation in response to ethylene induction, the processes governing FMI and floral morphogenesis, which require the action of several FMI genes and floral development genes, including FLOWERING LOCUS T (FT), LEAFY (LFY) and PISTILLATA $(P I)$, were investigated $[12,18]$. The pineapple expression of the $F T$ and PI genes reached the highest level at 40 days after floral induction, at which time multiple fruit and floral organs were forming; this phenomenon proved the important role of AcFT and AcPI in the development of floral organs [26-27]. Liu and Fan also reported that the pineapple FTlike and AP1-like (APETALA1) genes, as well as the CAL-like (CAULIFLOWER) and AG-like (AGAMOUS) genes, were upregulated 50 days after ethephon stimulation [18].

Despite its success and economic advantages in pineapple, ethephon has never been entirely reliable, and partial or total failure of floral induction have often been reported 


\section{Cellular Physiology Cell Physiol Biochem 2018;50:2139-2156 \\ \begin{tabular}{l|l|l} 
and Biochemistry Published online: 10 November 2018 & $\begin{array}{l}\text { ○ } 2018 \text { The Author(s). Published by S. Karger AG, Basel } \\
\text { www.karger.com/cpb }\end{array}$ \\
\hline
\end{tabular}}

Liu et al.: Comparative Transcriptome and Proteome Analyses of Ethephon-Induced

Pineapple Floral Transition

[28]. The role of ethylene in floral induction is controversial and varies due to the difference in pineapple cultivars categorized [29]. It is easy for the flowering of pineapple plants categorized as Queen to be induced by ethephon, whereas this floral induction is difficult or even completely ineffective for those categorized as Cayenne, such as 'Smooth Cayenne' (SC), as well as for some spineless hybrid pineapple cultivars such as MD-2, Tainong 17 and Tainong $18[14,30]$. For those categorized as Cayenne, only a portion of ethephon-treated buds are competent enough to complete the phase transition from vegetative to reproductive growth, while others remain in vegetative growth, indicating the ability to revert back to vegetative growth (Fig. S1 - for all supplemental material see www.karger.com/10.1159/000495057). Flowering failures not only reduce yields but also affect fruit quality and cause harvest problems, which hinders production.

Little information is available regarding the role of ethephon stimulation for different categories of pineapple species and the mechanisms underlying the aforementioned issues. Accordingly, in this study, comparative analyses of the transcriptome and proteome of the two types of pineapple samples (categorized as either Queen or Cayenne) were performed for control (CK) and ethephon-treated plants to identify the important regulators and metabolic pathway variations involved. This study is expected to clarify two major issues: 1 ) what the basic differences in the transcriptomic and proteomic levels between the two different types of pineapple are before ethephon induction treatment and 2) what the variations in the DEGs and DEPs (differentially expressed proteins) between the two different types of pineapple plants responding to ethephon induction are as well as the metabolic pathways involved. The findings of this work contribute to the understanding of the molecular regulatory mechanisms of the ethephon-mediated stimulation of the floral transition in the two types of pineapple plants that differ in their floral induction behavior.

\section{Materials and Methods}

\section{Plant materials and treatments}

To investigate the variation in the floral transition between the two types of pineapple plants in response to ethephon-mediated induction, the pineapple cultivars 'Comte de Paris' and 'Smooth Cayenne', which are categorized as Queen and Cayenne, respectively, were used as materials. To easily distinguish the two cultivars in the present study, they are referred to as CP and SC, respectively. Forty-eight plants of both CP and SC (ninety-six in total) were selected for ethephon induction experiments; the selected plants displayed a similar height and number of leaves. On October $12^{\text {th }}, 2016$, half of the plants were treated with $100 \mathrm{~mL}$ of ethylene solution containing $2.40 \mathrm{~mL} \cdot \mathrm{L}^{-1}$ ethephon $(\mathrm{v} / \mathrm{v}, 40 \%)$, which was applied to the central cup. The ethephon-treated CP and SC plants are referred to as CP-T and SC-T, respectively. The other plants were treated with the same volume of clean water and used as CKs. The CP and SC CK plants are referred to as $\mathrm{CP}-\mathrm{CK}$ and $\mathrm{SC}-\mathrm{CK}$, respectively.

Eight days later, nine shoot apical meristems $(9 \times 4=36$ total $)$ were randomly sampled from the CP-T, SC-T, CP-CK and SC-CK groups. All of the samples were immediately submerged in liquid nitrogen. Of the nine shoot apical meristems from each treatment and the CK treatment, six were used for transcriptomic analyses, and three were used for proteomic analyses. With respect to the transcriptomic analyses, the six shoot apical meristems from each treatment and the CK treatment were equally divided into two groups as two biological replicates.

\section{RNA extraction, library construction and sequencing}

The total RNA was extracted from each sample using a Trizol kit (Promega, USA) in accordance with the manufacturer's illustrations. Afterward, the total RNA was treated with RNase-free DNase I (TakaraBio, Japan) for $30 \mathrm{~min}$ at $37^{\circ} \mathrm{C}$ to remove any residual DNA. The RNA quality was verified using a bioanalyzer (Agilent 2100 Technologies, Santa Clara, CA) and was also evaluated by RNase-free agarose gel electrophoresis. The concentration of the total RNA was measured at 260 and $280 \mathrm{~nm}$ using a bioanalyzer.

The mRNAs were subsequently enriched using oligo (dT) magnetic beads (Qiagen) and then broken down into short fragments. First-strand cDNA was synthesized using random hexamer primers. Second- 


\section{Cellular Physiology Cell Physiol Biochem 2018;50:2139-2156 \begin{tabular}{l|l|l|l}
\hline DOI: 10.1159/000495057 & $\begin{array}{l}\text { C) } 2018 \text { The Author(s). Published by S. Karger AG, Basel } \\
\text { www.karger com/cpb }\end{array}$
\end{tabular} and Biochemistry \\ Liu et al.: Comparative Transcriptome and Proteome Analyses of Ethephon-Induced}

Pineapple Floral Transition

strand cDNA was subsequently generated using RNase $\mathrm{H}$ and DNA polymerase I. After purification, end reparation and poly (A) addition, sequencing adapters were ligated to the cDNA. The cDNA was then purified via agarose gel electrophoresis and enriched by PCR amplification to generate a final cDNA library. Afterward, the cDNA libraries were sequenced on an Illumina HiSeq ${ }^{\text {TM }} 2000$ platform using paired-end technology. Clean reads were selected by removing any low-quality reads, adaptors containing reads, and reads containing $>10 \% \mathrm{~N}$ bases.

\section{DEGs and functional enrichment analyses}

The clean RNA-seq reads were mapped to a pineapple reference genome [31] by TopHat 2 . The normalized transcript abundance of the genes was calculated using the fragments per kilobase of transcript per million mapped reads (FPKM) method, and subsequently, differential expression analysis was performed to determine the threshold p-value, false discovery rate (FDR) and fold change ( $\log _{2}$ ratio) from two biological replicates using the edgeR package (http://www.r-project.org/). DEGs were defined as those with an FDR $\leq 0.05$ and an absolute value of $\log _{2}$ ratio $\geq 1$. The DEGs were used for GO and KEGG enrichment analyses in accordance with the methods described by Kanehisa et al. [32] and Ashburner et al. [33], respectively. The GO terms and KEGG pathways with $P$-values $\leq 0.05$ were considered significantly enriched in DEGs.

\section{Validation of DEG subsets by $q P C R$}

Nineteen DEGs were subjected to qPCR to validate the accuracy and reproducibility of the transcriptome analysis RNA-seq results. The gene expression levels were determined using the Ct values with the formula $2^{-\triangle \mathrm{Ct}}$. The actin gene of $A$. comosus (HQ148720.1) was used as a reference gene. Each qPCR analysis was performed in two biological replicates and three technical replicates. The primer sequences are shown in Table S1.

\section{Protein extraction}

The total proteins were extracted using the cold acetone method. Samples were ground to a powder in liquid nitrogen and then dissolved in $2 \mathrm{~mL}$ of lysis buffer [8 $\mathrm{M}$ urea, 2\% SDS, 1X protease inhibitor cocktail (Roche Ltd. Basel, Switzerland)], followed by sonication on ice for $30 \mathrm{~min}$ and centrifugation at 13, 000 $\mathrm{rpm}$ at $4{ }^{\circ} \mathrm{C}$ for $30 \mathrm{~min}$. The supernatant was transferred to a clean tube, after which the proteins were precipitated with ice-cold acetone at $-20{ }^{\circ} \mathrm{C}$ overnight. The precipitations were rinsed with acetone three times and then redissolved with $8 \mathrm{M}$ urea by sonication on ice. The protein quality was examined with SDSPAGE in accordance with the manufacturer's instructions. A bicinchoninic acid assay (BCA) protein assay kit was used to determine the protein concentration of the supernatant.

Protein digestion and isobaric tag for relative and absolute quantitation (iTRAQ)/tandem mass tag (TMT) labeling

Proteins were tryptic digested with sequence-grade modified trypsin (Promega, Madison, WI) at $37^{\circ} \mathrm{C}$ overnight. The digested samples were then centrifuged at 13, $500 \mathrm{rpm}$ for $12 \mathrm{~min}$, dried under vacuum, and dissolved in $500 \mathrm{mM}$ tetraethylammonium bromide (TEAB). The resultant peptide mixture was labeled with iTRAQ/TMT tags [iTRAQ Reagents-8Plex (SCIEX)] for 2 hours at room temperature. The labeled samples were then combined and dried under vacuum.

\section{HPLC-MS/MS analysis and database search}

A fusion mass spectrometer was operated in data-dependent acquisition mode to switch automatically between MS and MS/MS acquisition. Full-scan MS spectra (m/z 350-1550) were acquired at a mass resolution of $120 \mathrm{~K}$, followed by sequential high-energy collisional dissociation (HCD) MS/MS scans at a resolution of $30 \mathrm{~K}$. The isolation window was set as $1.6 \mathrm{Da}$. The AGC target was set as 400, 000. The MS/MS fixed first mass was set at 110. In all cases, one microscan was recorded using a dynamic exclusion of 45 seconds.

The tandem mass spectra were extracted, after which the charge state was deconvoluted and deisotoped by Mascot Distiller version 2.6. The MS data were then transformed into MGF files with Proteome Discovery version 1.2 (Thermo, Pittsburgh, PA, USA) and analyzed using a Mascot search engine (Matrix Science, London, UK; version 2.3.2). The Mascot database was set up for protein identification using the pineapple reference transcriptome database [31]. Mascot was queried with a fragment ion mass tolerance of $0.050 \mathrm{Da}$ 


\section{Cellular Physiology and Biochemistry}

Cell Physiol Biochem 2018:50:2139-2156

\begin{tabular}{l|c|c|}
\hline DOI: 10.1159/000495057 & C 2018 The Author(s). Published by S. Karger AG, Basel
\end{tabular}

Published online: 10 November 2018 www.karger.com/cpb

Liu et al.: Comparative Transcriptome and Proteome Analyses of Ethephon-Induced

Pineapple Floral Transition

and a parent ion tolerance of $20.0 \mathrm{ppm}$. Carbamidomethyl of cysteine as well as iTRAQ8plex of lysine and the N-terminus were specified in Mascot as fixed modifications. Deamidation of asparagine and glutamine, oxidation of methionine and acetylation of the $\mathrm{N}$-terminus were specified as variable modifications in Mascot.

Protein identification and quantification

Protein identification was accepted if the protein could present a FDR of less than $1.0 \%$ according to the scaffold local FDR algorithm. The proteins that contained similar peptides and could not be differentiated based on MS/MS analysis alone were grouped together to satisfy the principles of parsimony.

Proteins were quantified in all the identified samples with a unique spectra $\geq 2$. The Mascot search results were averaged via medians and then quantified. The proteins whose fold change in expression within a comparison was $>1.2$ or $<0.83$ and whose unadjusted significance level ( $p$-value) was less than 0.05 were considered differentially expressed.

Protein functional annotation and enrichment analyses

To identify their functions, the proteins were annotated against the GO and KEGG databases. Significant GO functions and pathways were examined for DEPs with a $P$-value $\leq 0.05$.

Protein and RNA correlation analyses

To compare the agreement between transcriptomic changes and proteomic changes during the ethephon-induced floral induction process, correlations were analyzed based on the DEPs and DEGs. Pearson correlation tests were conducted for each comparison group, including CP-CK vs SC-CK, CP-T vs SC-T, SC-T vs SC-CK and CP-T vs SC-T.

\section{Results}

Basic differences at the transcriptomic and proteomic levels between CP and SC before ethephon treatment

Before the ethephon treatment, in the comparison between CP-CK and SC-CK, 1, 633 DEGs were identified, including 504 upregulated and 1, 129 downregulated genes (Fig. 1A). In the comparison between CP-CK and SC-CK, with respect to protein expression, 114 DEPs were identified, including 62 upregulated and 52 downregulated proteins (Fig. 1A).

Furthermore, the DEGs ETR, ERF, ACS and ACO were identified in the CP-CK and SC-CK samples (Table 1). Among these genes, the expression of ETR (Aco024515) in CP-CK was lower than that in SC-CK by 2.19-fold. The ERF (Aco017803) gene was expressed at a lower level in CP-CK than in SC-CK by 4.18-fold. The expression levels of the ACS (Aco015869) and ACO (Aco001358) genes in CP-CK were higher than those in SC-CK.

The ACO protein was identified as a DEP in the comparison between CP-CK and SC-CK, and its expression was lower in CP-CK than in SC-CK (Table 1).

DEGs and DEPs related to "response to ethephon induction" between CP and SC

After the ethephon treatment, a total of 2, 984 DEGs were identified between CP and SC (by comparing the groups of CP-T and SC-T) (Fig. 1A). Among these 2,984 DEGs, 1,354 genes were upregulated, and 1,630 were downregulated. Similarly, compared with those in SC-T, a total of 260 DEPs in CP-T were identified after ethephon induction, including 94 upregulated and 166 downregulated proteins (Fig. 1A).

Within a cultivar, the transcriptomic and proteomic changes involved in the pineapple floral transition in response to ethephon-inductive cues were further explored by comparing the CP-T and CP-CK and SC-T and SC-CK groups (Fig. 1B). A total of 782 DEGs were identified in the comparison between CP-T and CP-CK, including 514 upregulated and 268 downregulated genes. Similarly, a total of 1,352 DEGs were identified in the comparison between SC-T and 


\section{Cellular Physiology Cell Physiol Biochem 2018;50:2139-2156 and Biochemistry DOl: 10.1159/000495057 2018 (0) 2018 The Author(s). Published by S. Karger AG, Basel \\ Liu et al.: Comparative Transcriptome and Proteome Analyses of Ethephon-Induced \\ Pineapple Floral Transition}

Fig. 1. Number of DEGs and DEPs in each comparison. (A) Indicated are the numbers of DEGs and DEPs between the $\mathrm{CP}$ and SC cultivars before and after ethephon treatment. The red columns show the numbers of upregulated genes and proteins in the comparison between $\mathrm{CP}$ and SC (the expression values of CP compared to SC), and the blue columns show the numbers of downregulated genes and proteins. (B) Indicated are the numbers of DEGs and DEPs affected by ethephon treatment within a cultivar. The red columns show the numbers of upregulated genes and proteins in CP or SC after ethephon treatment in comparison with those before treatment, respectively. The blue columns show the numbers of downregulated genes and proteins.

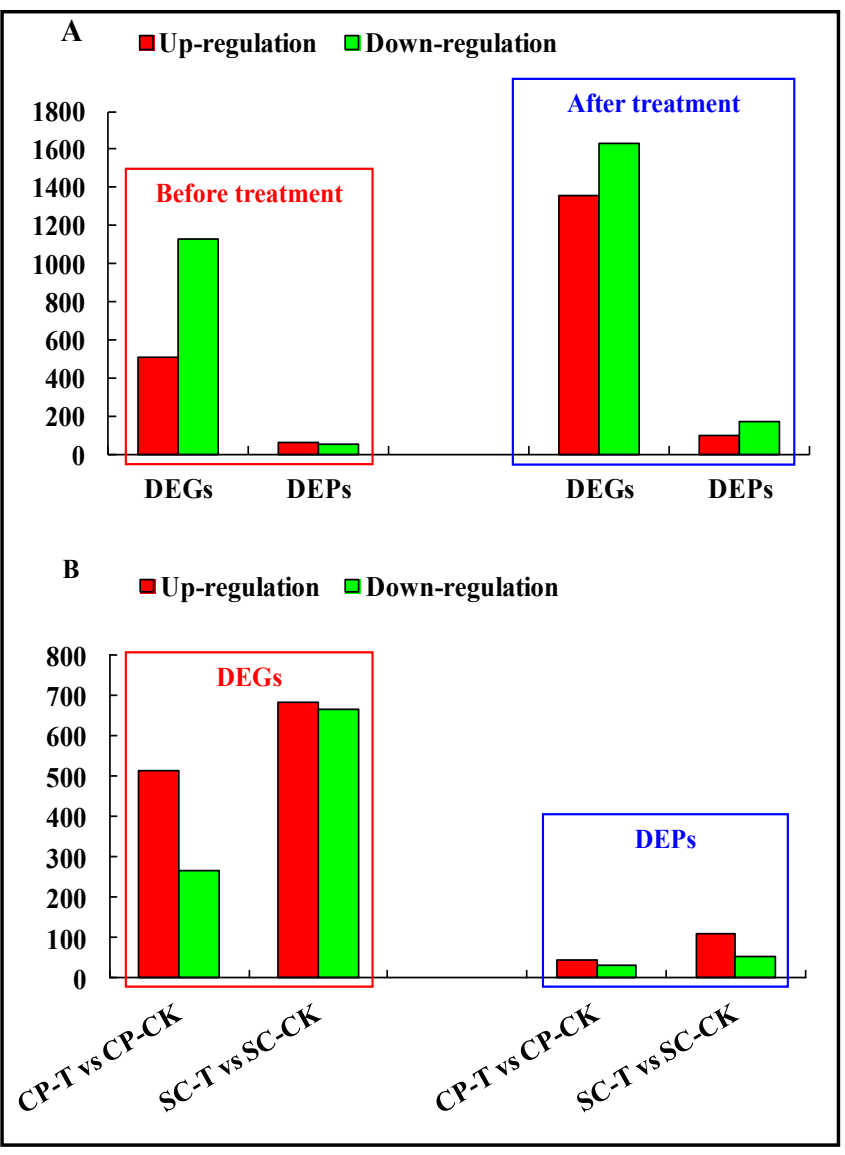

Table 1. Basic differences in the ethylene signaling genes between $\mathrm{CP}$ and SC before ethephon treatment. * The fold change indicates the up- or down-regulated changes in expression in CP compared with that in SC. The gene fold changes indicate the $\log _{2}{ }^{\text {Ratio, }}$, the protein fold change directly indicates the ratio; the same for the next tables

\begin{tabular}{lccc}
\hline ID & Name & Description & Fold changes* \\
\hline Aco024515 & ETR gene & Ethylene receptor & -2.19 \\
Aco017803 & ERF gene & Ethylene-responsive transcription factor & -4.18 \\
Aco015869 & ACS gene & 1-aminocyclopropane-1-carboxylate synthase & 2.92 \\
Aco001358 & ACO gene & 1-aminocyclopropane-1-carboxylate oxidase & 1.39 \\
Aco015240 & ACO protein & 1-aminocyclopropane-1-carboxylate oxidase & 0.83 \\
\hline
\end{tabular}

SC-CK. Among them, 685 were upregulated, and 667 were downregulated. With respect to proteins, compared with those in CP-CK, a total of 78 DEPs in CP-T were identified, including 44 upregulated and 34 downregulated proteins. Similarly, a total of 163 DEPs were identified in the comparison group between SC-T and SC-CK. Among them, 110 were upregulated, and 53 were downregulated.

GO analysis of the DEGs and DEPs related to "response to ethephon induction" between CP and $S C$

To investigate the molecular changes and biological functions of the DEGs and DEPs during the ethephon induction process, GO terms belonging to the biological process (BP) and molecular function (MF) categories were identified. As highlighted in Fig. 2, the GO terms for the CP-T and CP-CK and SC-T and SC-CK comparison groups differed. 
Liu et al.: Comparative Transcriptome and Proteome Analyses of Ethephon-Induced

Pineapple Floral Transition

A

口CP-T vs CP-CK $\square$ SC-Tvs SC-CK

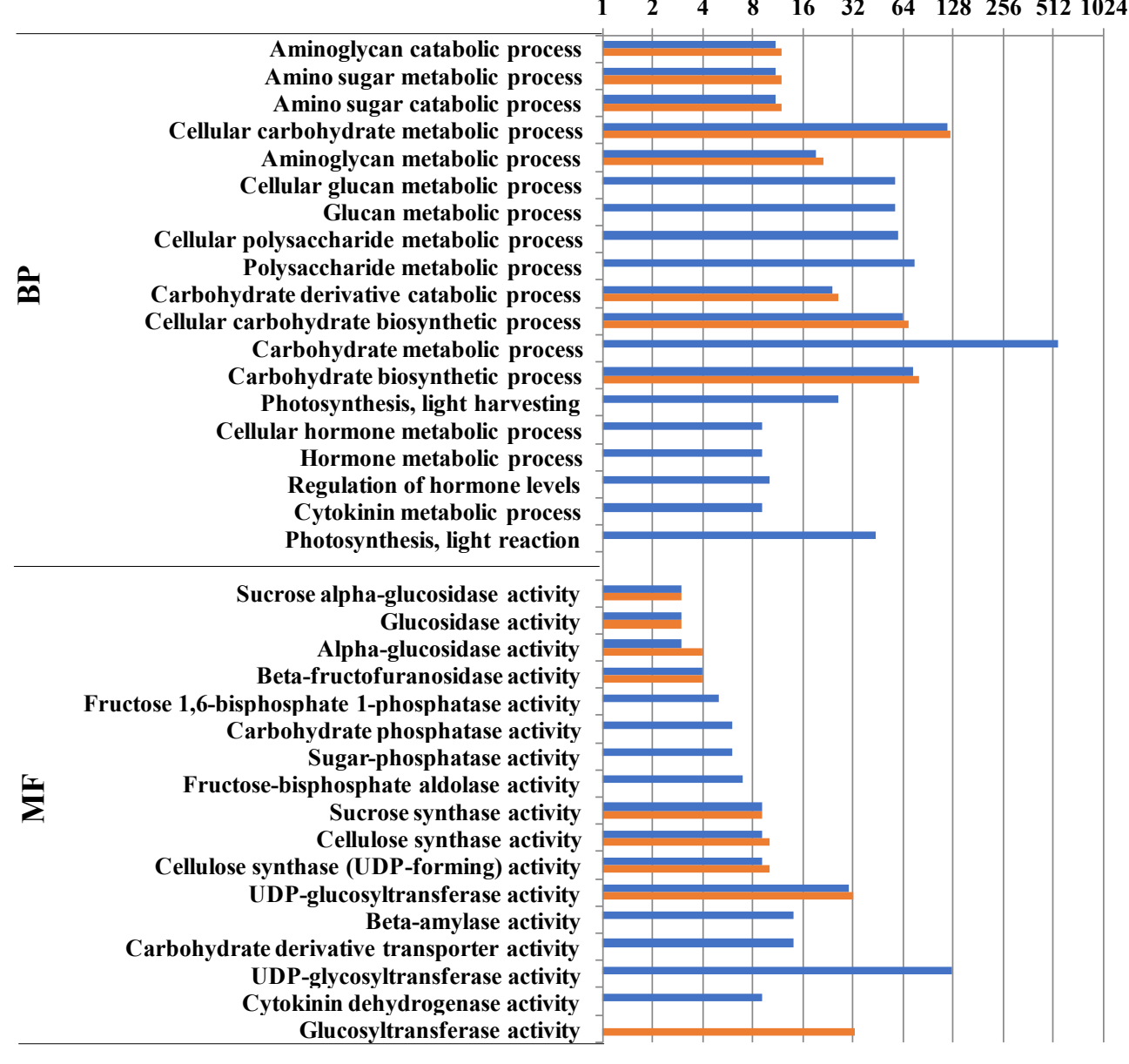

"CP-T vs CP-CK $\square$ SC-Tvs SC-CK

B

$\begin{array}{lll}1 & 4 & 16\end{array}$

64

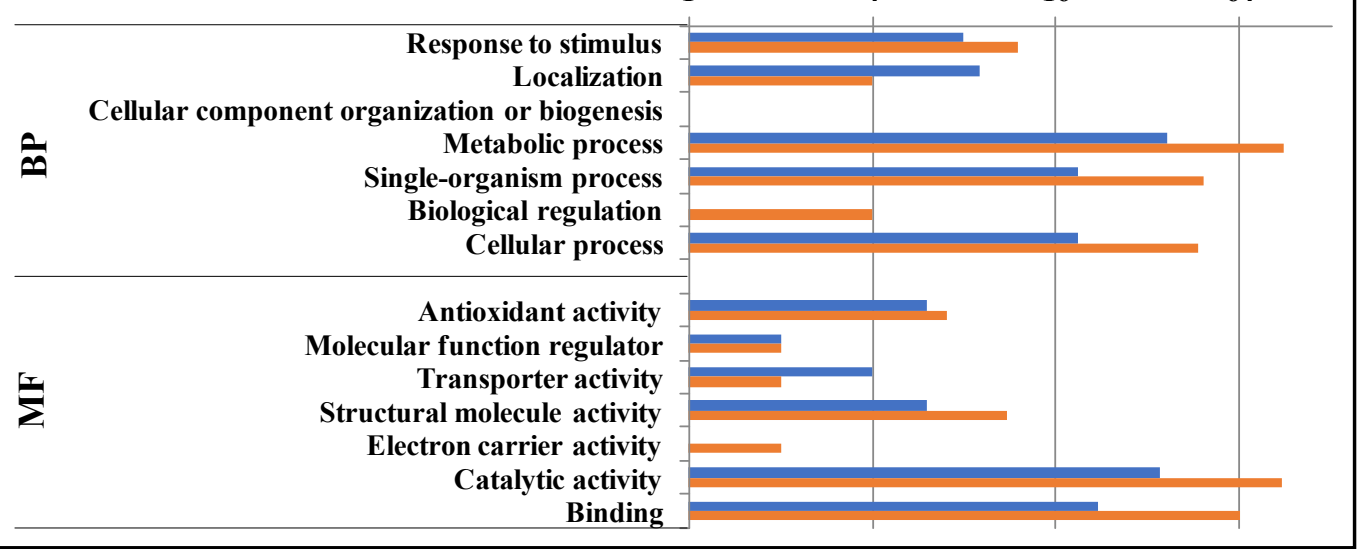

Fig. 2. GO classifications of the DEGs (A) and DEPs (B) in the CP-T and CP-CK and the SC-T and SC-CK comparison groups. 
Liu et al.: Comparative Transcriptome and Proteome Analyses of Ethephon-Induced

With respect to genes, in the comparison group between CP-T and CP-CK, the DEGs were predominantly annotated to $19 \mathrm{GO}$ terms in the BP category. These annotated GO terms, which included carbohydrate metabolic process (GO:0005975), cellular carbohydrate metabolic process (GO:0044262), cellular hormone metabolic process (G0:0034754) and regulation of hormone levels (GO:0010817), were involved predominantly in carbohydrate metabolism, plant hormone signal transduction and metabolism. Additionally, the GO terms associated with photosynthesis and photoperiod, which included the photosynthesis and light reaction (G0:0019684) as well as the photosynthesis and light harvesting (GO:0009765) terms, were enriched in the comparison between CP-T and CP-CK. In the MF category, the DEGs were predominantly annotated to $16 \mathrm{GO}$ terms, including UDP-glycosyltransferase activity (G0:0008194), UDP-glucosyltransferase activity (GO:0035251), sucrose synthase activity (G0:0016157) and cellulose synthase activity (GO:0016759); these terms were involved predominantly in carbohydrate metabolism. Additionally, the plant hormone transductionassociated GO term cytokinin dehydrogenase activity (GO:0019139) was enriched in CP-T and CP-CK. In the comparison group between SC-T and SC-CK, the DEGs were predominantly annotated to $8 \mathrm{GO}$ terms in the BP category. These GO terms were involved predominantly in carbohydrate/sugar metabolism and included cellular carbohydrate metabolic process (G0:0044262), cellular carbohydrate biosynthetic process (G0:0034637) and carbohydrate biosynthetic process (G0:0016051) terms. No GO terms associated with plant hormone signal transduction or photosynthesis and photoperiod were enriched with DEGs in the comparison between SC-T and SC-CK. In the MF category for SC-T and SC-CK, the DEGs were predominantly annotated to 9 GO terms, including UDP-glucosyltransferase activity (G0:0035251), glucosyltransferase activity (G0:0046527) and cellulose synthase activity (GO:0016759), which were involved predominantly in carbohydrate metabolism.

With respect to proteins, in the CP-T and CP-CK comparison group, the DEPs were annotated predominantly to the GO terms of metabolic process (GO:0008152), singleorganism process (GO:0044699), cellular process (GO:0009987) and response to stimulus (G0:0050896) in the BP category and catalytic activity (GO:0003824) and binding (GO:0005488) in the MF category. In the SC-T and SC-CK comparison group, the annotation of the DEPs was similar to that in the CP-T and CP-CK comparison group, and GO terms including metabolic process, single-organism process, cellular process and response to stimulus in the BP category as well as catalytic activity and binding in the MF category were identified.

KEGG analysis of the DEGs and DEPs related to "response to ethephon induction" between $C P$ and $S C$

A KEGG pathway analysis was performed to further examine the DEGs and DEPs (Fig. 3 ). With respect to the genes, in the comparison between CP-T and CP-CK, many DEGs were enriched in 11 metabolic pathways, including carbohydrate metabolism pathways such as starch and sucrose metabolism (ko00500), amino sugar and nucleotide sugar metabolism (ko00520), and pentose and glucuronate interconversions (ko00040); plant hormone signal transduction pathways such plant hormone signal transduction (ko04075), brassinosteroid biosynthesis (ko00905) and ZT biosynthesis (ko00908); and plant photoperiod pathways such as those of photosynthesis antenna proteins (ko00196) and the circadian rhythmplant (ko04712). In the comparison between SC-T and SC-CK, many DEGs were enriched in 5 metabolic pathways including galactose metabolism (ko00052), plant hormone signal transduction (ko04075) and amino sugar and nucleotide sugar metabolism (ko00520).

With respect to proteins, in the comparison between CP-T and CP-CK, many DEPs were enriched in starch and sucrose metabolism (ko00500), carbon metabolism (ko01200), glyoxylate and dicarboxylate metabolism (ko00630), the citrate cycle (tricarboxylic acid (TCA) cycle) (ko00020), amino sugar and nucleotide sugar metabolism (ko00520), and glycolysis/gluconeogenesis (ko00010). Similar results were obtained in the DEPs of the SC-T and SC-CK comparison group. 
Liu et al.: Comparative Transcriptome and Proteome Analyses of Ethephon-Induced

Pineapple Floral Transition

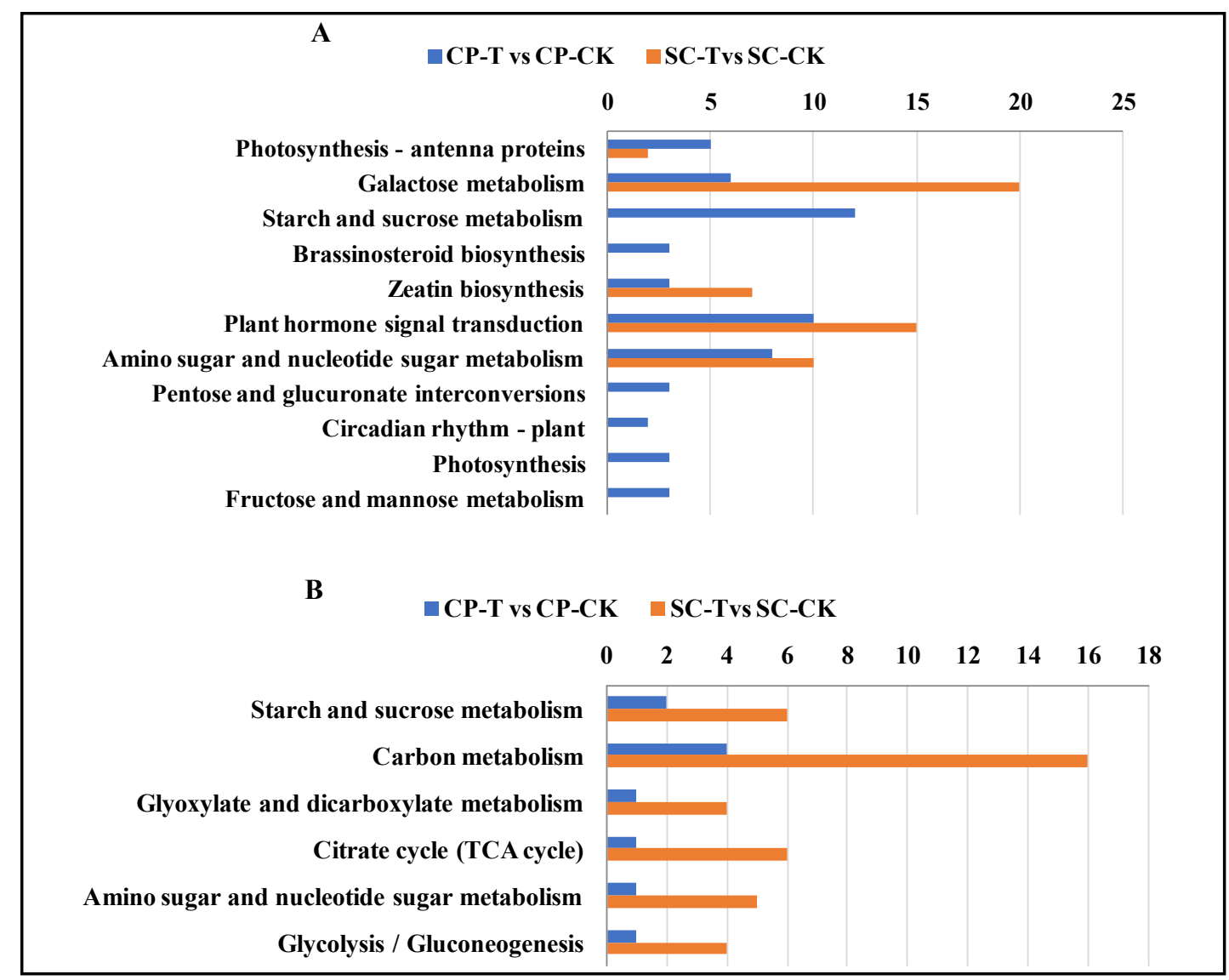

Fig. 3. KEGG pathways of the DEGs (A) and DEPs (B) in the CP-T and CP-CK and the SC-T and SC-CK comparison groups.

Variation in genes related to floral induction between CP and SC in response to ethephon induction

The DEGs related to floral transition in response to ethephon induction in pineapple were identified, and the differential expression of these genes in ethephon-treated CP and SC were compared. Tables 2 and 3 summarize the expression of these ethephon inductionrelated DEGs.

\section{ERFs and ACO}

In the comparison between CP-T and CP-CK, five ERFs, namely, Aco017803, Aco001844, Aco012860, Aco001600, and Aco009511, were identified as DEGs and were found to be upregulated by 7.12-, 2.85-, 2.33-, 1.21- and 1.09-fold, respectively. In the comparison between SC-T and SC-CK, three ERFs, namely, Aco017803, Aco001844 and Aco012860, were identified as DEGs and were upregulated by 4.00-, 1.61- and 1.60-fold, respectively.

In the comparison between CP-T and CP-CK, one ACO gene, Aco015240, was identified as being differentially expressed and was upregulated by 1.52-fold in CP-T. In the comparison between SC-T and SC-CK, the ACO Aco015240 gene was upregulated by 1.30 -fold in SC-T.

\section{Genes related to carbohydrate metabolism}

In the CP-T and CP-CKcomparison, the SPS (sucrose phosphate synthase) gene Aco017378, the $B A M$ (beta-amylase) gene Aco014607 and the FBP (fructose-1, 6-bisphosphatase) gene Aco016862 were upregulated by 2.89-, 2.00- and 1.88-fold, respectively, in CP-T. With the exception of $F B P$, these genes were identified as DEGs in the comparison between SC-T and SC-CK, and their expression levels were upregulated by 2.30 - and 1.05-fold, respectively, in SC-T. 
Liu et al.: Comparative Transcriptome and Proteome Analyses of Ethephon-Induced

Pineapple Floral Transition

Table 2. A list of some DEGs detected in the CP-T and CPCK and the SC-T and SC-CK comparison groups

\begin{tabular}{lccc}
\hline \multirow{2}{*}{ ID } & Description & \multicolumn{2}{c}{ Fold changes } \\
& CP-T vs CP-CK & SC-T vs SC-CK \\
\hline \multicolumn{2}{c}{ Ethylene-related genes } \\
Aco017803 & ERF (ethylene-responsive factor) & 7.12 & 4.00 \\
Aco001844 & ERF & 2.85 & 1.61 \\
Aco012860 & ERF & 2.33 & 1.60 \\
Aco001600 & ERF & 1.21 & - \\
Aco009511 & ERF & 1.09 & - \\
Aco015240 & ACO (ACC oxidase) & 1.52 & 1.30 \\
Carbohydrate & metabolism associated genes & & \\
Aco017378 & SPS (sucrose phosphate synthase) & 2.89 & 2.30 \\
Aco014607 & BAM (beta-amylase) & 2.00 & 1.05 \\
Aco016862 & FBP (fructose-1,6-bisphosphatase) & 1.88 & - \\
FMI-related genes & & & \\
Aco004839 & AGL8 (AGMOUS-like) & 11.69 & 10.10 \\
Aco012428 & AGL8 & 9.86 & 4.69 \\
Aco017563 & AGL4 & 4.20 & - \\
Aco015104 & AGL10 & 1.38 & - \\
Aco016718 & TFL (TERMINAL FLOWER) & -2.54 & -1.69 \\
Aco003470 & FT (FLOWERING LOCUS T) & 8.95 & 3.46 \\
\hline
\end{tabular}

\section{$A G$}

In the comparison between CP-T and CP-CK, four AGs (Aco004839, Aco012428, Aco017563 and Aco015104) were identified as DEGs and were found to be upregulated by 11.69-, 9.86-, 4.20-, and 1.38-fold, respectively, in CP$\mathrm{T}$. In the comparison between SC-T and SC-CK, only two corresponding genes, Aco004839 and Aco012428, were identified as DEGs and were upregulated by 10.10 - and 4.69-fold, respectively, in SC-T.

\section{TFL}

In the comparison between CP-T and CPCK, one TFL gene, Aco016718, was identified as a DEG and was downregulated by 2.54 -fold in CP-T, whereas Aco016718 was downregulated by 1.69 -fold in SC-T in the comparison between SC-T and SC-CK.

\section{FT}

In the CP-T and CP-CK and SC-T and SC-CK comparison groups, the FT gene Aco003470 was found to be upregulated by 8.95- and 3.46-fold in CP-T and SC-T, respectively.
Table 3. A list of some DEGs detected in the CP-T and CP-CK and the SC-T and SC-CK comparison groups

\begin{tabular}{|c|c|c|c|}
\hline \multirow[b]{2}{*}{ ID } & \multirow[b]{2}{*}{ Description } & \multicolumn{2}{|c|}{ Fold changes } \\
\hline & & $\begin{array}{l}\text { CP-T vs } \\
\text { CP-CK }\end{array}$ & $\begin{array}{l}\text { SC-T vs } \\
\text { SC-CK }\end{array}$ \\
\hline Aco011242 & MYB & 2.27 & - \\
\hline Aco010021 & MYB & 2.18 & 1.42 \\
\hline Aco002526 & MYB & 2.05 & 1.94 \\
\hline XLOC_017829 & MYB & 1.64 & - \\
\hline Aco027180 & MYB & 1.63 & - \\
\hline Aco007733 & MYB & - & 10.23 \\
\hline XLOC_023363 & MYB & - & 4.85 \\
\hline Aco000681 & MYB & - & 4.00 \\
\hline Aco001748 & MYB & - & 3.80 \\
\hline Aco002582 & MYB & - & 2.00 \\
\hline Aco005966 & MYB & - & 1.55 \\
\hline Aco030108 & MYB & - & 1.31 \\
\hline Aco020986 & MYB & - & 1.30 \\
\hline Aco017254 & MYB & - & 1.26 \\
\hline Aco009947 & MYB & - & 1.23 \\
\hline Aco009969 & MYB & - & 1.00 \\
\hline Aco008200 & WRKY & 2.99 & 3.73 \\
\hline Aco028684 & WRKY & 2.60 & - \\
\hline Aco008276 & WRKY & 1.95 & - \\
\hline Aco016066 & WRKY & 1.58 & 1.09 \\
\hline Aco005341 & WRKY & 1.26 & 1.75 \\
\hline Aco001643 & WRKY & 1.21 & 1.17 \\
\hline Aco002567 & WRKY & 1.05 & 1.50 \\
\hline Aco022888 & WRKY & - & 3.86 \\
\hline Aco027950 & WRKY & - & 3.38 \\
\hline Aco011109 & WRKY & - & 3.36 \\
\hline Aco005587 & WRKY & - & 3.27 \\
\hline Aco022106 & WRKY & - & 2.81 \\
\hline Aco006221 & WRKY & - & 2.01 \\
\hline Aco012598 & NAC & 4.63 & 4.64 \\
\hline Aco019379 & NAC & - & 6.82 \\
\hline Aco023285 & NAC & - & 3.60 \\
\hline Aco012922 & NAC & - & 2.77 \\
\hline Aco011855 & bHLH & 3.41 & 2.28 \\
\hline Aco009100 & bHLH & 3.09 & - \\
\hline Aco001133 & bHLH & 1.91 & 1.64 \\
\hline Aco011419 & bHLH & 1.85 & - \\
\hline Aco010917 & bHLH & 1.46 & 1.52 \\
\hline Aco014894 & bHLH & 1.27 & - \\
\hline Aco003613 & bHLH & 1.21 & - \\
\hline Aco022096 & bHLH & 1.05 & - \\
\hline Aco002246 & bHLH & - & 1.76 \\
\hline
\end{tabular}




\section{Cellular Physiology Cell Physiol Biochem 2018;50:2139-2156

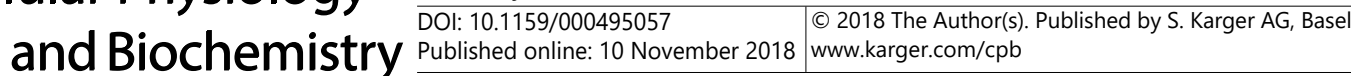 Liu et al.: Comparative Transcriptome and Proteome Analyses of Ethephon-Induced \\ Pineapple Floral Transition}

$M Y B, W R K Y, N A C$ and $b H L H$ TFs

Several differentially expressed TFs such as MYBs, WRKYs, NACs and bHLHs were identified in the CP-T and CP-CK and SC-T and SC-CK comparison groups (Table 3). In the comparison between CP-T and CP-CK, 5 MYBs, 7 WRKYs, 1 NAC and 8 bHLHs were identified as DEGs and were consistently upregulated in CP-T. In the comparison between SC-T and SC-CK, these TFs constituted relatively more enriched DEGs. A total of $13 M Y B, 11$ WRKY, 4 $N A C$ and $4 \mathrm{bHLH}$ TFs were identified and were consistently upregulated. Most of these TFs were differentially expressed more in the comparison between SC-T and SC-CK than in the comparison between CP-T and CP-CK.

Variation in proteins related to floral induction between CP and SC in response to ethephon induction

The DEPs related to floral transition in response to ethephon induction in pineapple were identified. Table 4 summarizes the expression of these ethephon induction-related DEPs.

\section{ACO protein}

In the comparison between CP-T and CP-CK, the ACO protein (Aco001358), which was upregulated by 1.90 -fold in CP-T, was identified as a DEP. In the comparison between SC-T and SC-CK, Aco001358 was upregulated by 1.41-fold in SC-T.

\section{Proteins related to sugar metabolism}

In the comparison between CP-T and CP-CK, the glucose-1-phosphate adenylyltransferase (GluPAT) family protein (Aco006199) was identified as DEP and was upregulated by 1.64fold, in CP-T. Compared with that in CP-CK, the expression levels of two beta-glucosidase proteins (BGLUs), namely, Aco000757 and Aco000756 were upregulated by 1.67- and 1.52fold in CP-T, respectively. In the comparison between SC-T and SC-CK, none of previously mentioned proteins were identified.

Verification of gene expression data using qPCR

Nineteen of the identified DEGs were subjected to qPCR. Correlation analyses of the fold change ( $\log _{2}$ ratio) of the gene expression ratios revealed a significant positive correlation ( $R$ $=0.84463$ ) between the FPKM values obtained from the RNA-seq and qPCR analyses (Fig. 4).

Correlation analysis of RNA and protein expression

The tests revealed correlations ( $r$, Pearson) of $0.202,0.317,0.132$ and 0.565 , between the mRNA and protein ratios for the comparison groups CP-CK vs SC-CK, CP-T vs SC-T, CP-T vs CP-CK, and SC-T vs SC-CK, respectively (Fig. 5).

Table 4. A list of some DEPs detected in the CP-T and CP-CK and the SC-T and SC-CK comparison groups

\begin{tabular}{lccc}
\hline \multirow{2}{*}{ ID } & \multirow{2}{*}{ Description } & \multicolumn{2}{c}{ Fold changes } \\
& ACO (ACC oxidase) & CP-T vs CP-CK & SC-T vs SC-CK \\
\hline Aco001358 & GluPAT (glucose-1-phosphate adenylyltransferase) & 1.90 & 1.41 \\
Aco006199 & 1.64 & - \\
Aco000757 & BGLU (beta-glucosidase protein) & 1.67 & - \\
Aco000756 & BGLU & 1.52 & - \\
\hline
\end{tabular}




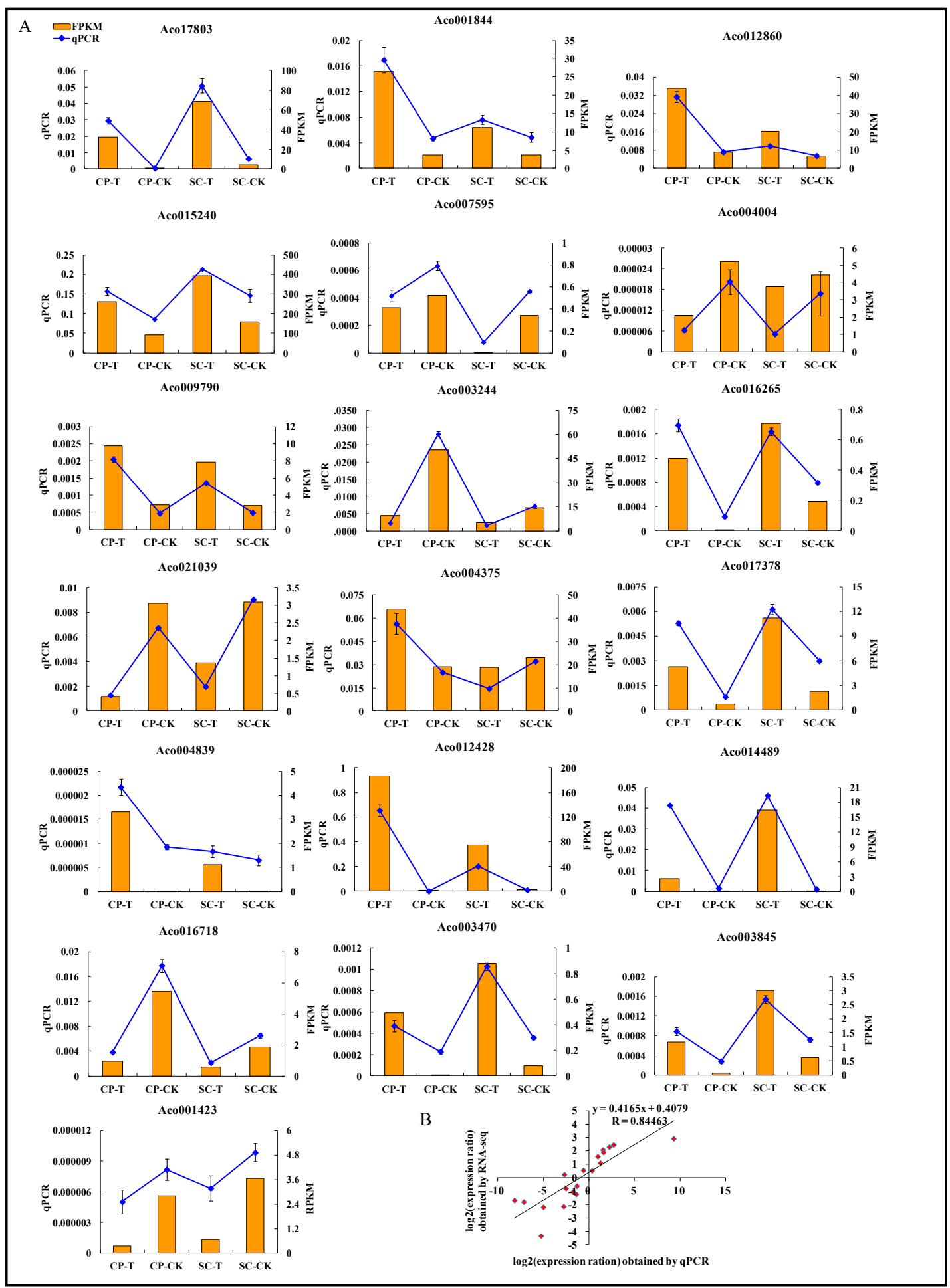

Fig. 4. qPCR validation of DEGs as determined by RNA-seq. (A) Nineteen DEGs were subjected to qPCR to validate the accuracy and reproducibility of the transcriptome analysis results of RNA-seq. The expression levels of the genes revealed by qPCR (left y-axis) and RNA-seq (right y-axis). The qPCR data were the means of two biological replicates and three technical replicates, and the bars represent the SE. The RNA-seq data are the means of two biological replicates. (B) The scatter plot was generated by the $\log _{2}$ expression ratios (T1/CK1 and T2/CK2) from RNA-seq ( $\mathrm{x}$-axis) and qPCR (y-axis). A significant correlation ( $\mathrm{R}=0.84463)$ was observed between the RNA-seq and qPCR data. 
Liu et al.: Comparative Transcriptome and Proteome Analyses of Ethephon-Induced

Pineapple Floral Transition

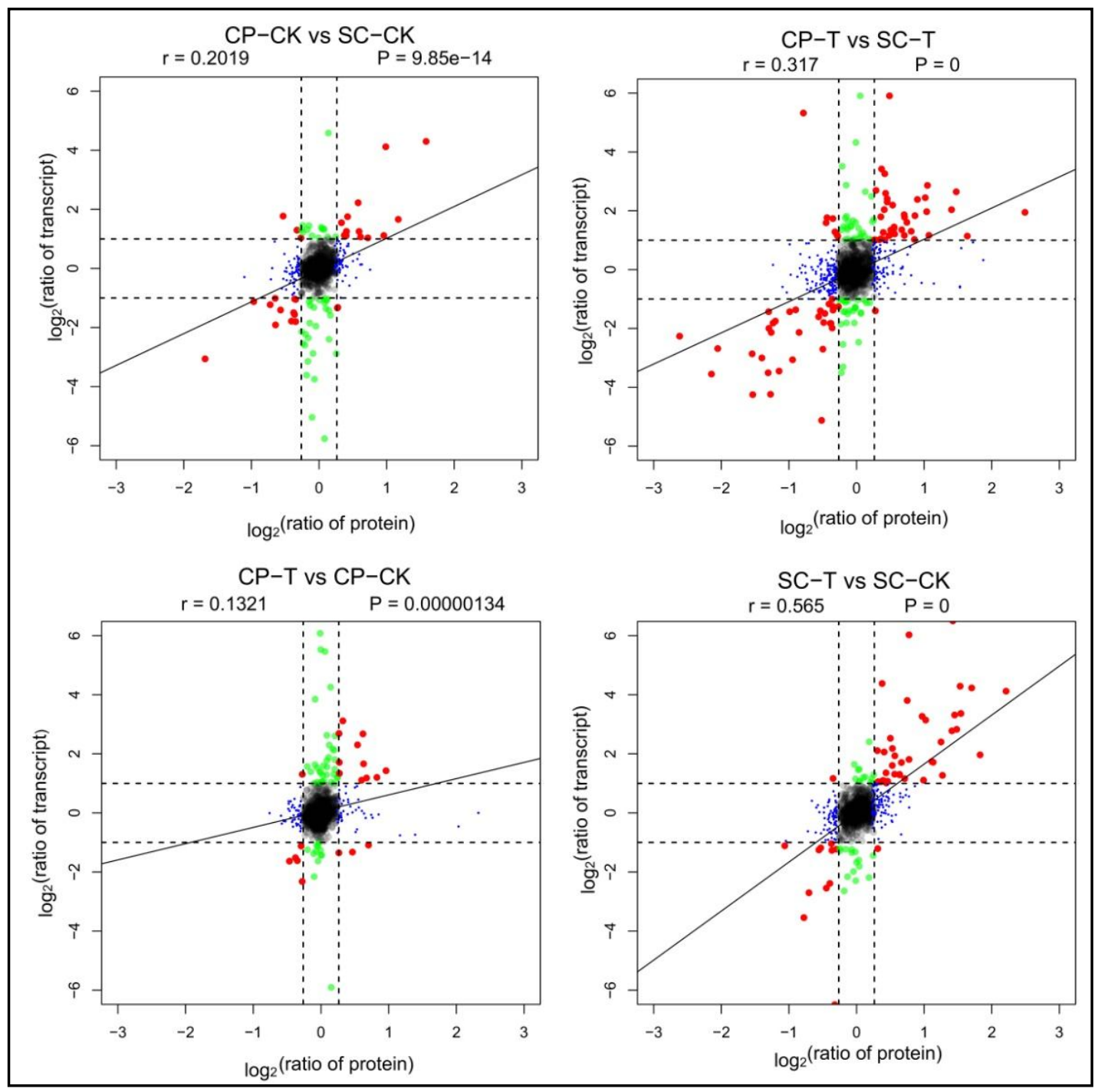

Fig. 5. Comparison of expression ratios from transcriptomic (y-axis) and proteomic ( $\mathrm{x}$-axis) profiling. The $\log _{2}$ (expression ratios) were calculated from the CP-CK and SC-CK, CP-T and SC-T, CP-T and CP-CK, and SC-T and SC-CK comparison groups. Significant changes in expression were labeled with color: blue, proteins only; green, transcripts only; red, both. Lines represent fitted straight trend lines from the data points.

\section{Discussion}

Variation in the basic ability of ethylene signal transduction may result in different responses to ethephon application between $C P$ and $S C$

Field culture practices have indicated that pineapple plants categorized as Cayenne usually continue their vegetative growth instead of transitioning to reproductive growth after ethephon stimulation, which differs from species categorized as Queen. In this work, many DEGs and DEPs were identified between the two types of pineapple, namely, CP and SC, which are categorized as Queen and Cayenne, respectively, before the ethephon induction treatment, which suggested that there are basic differences between CP and SC. Similar results occurred in the reports of the seasonal and continuous flowering roses in response to floral transition [34] as well as for low- and high-cadmium-accumulating genotypes of pakchoi (Brassica chinensis L.) in response to cadmium stress [35]. In particular, the results revealed that the expression levels of the ETR and ERF genes were lower in CP than in SC before the ethephon induction treatment, whereas those of the ACS and ACO genes were 
Liu et al.: Comparative Transcriptome and Proteome Analyses of Ethephon-Induced

Pineapple Floral Transition

higher. The DEP analysis also revealed that there was a difference between CP and SC with respect to ACO protein expression before ethephon induction treatment. ETR is a negative regulator of the ethylene signal transduction pathway, that is, it inhibits the pathway when it is not bound to ethylene [17]. Reductions in ETR and ERF levels increase the sensitivity of plants to ethylene, and a lower concentration of ethylene could stimulate the ethylene response and induce pineapple flowering [36-37]. The ACS and ACO genes are the key regulatory genes involved in the biosynthesis of ethylene $[23,38]$ and are upregulated by ethephon application when pineapple flowering is triggered $[12,23]$. Variation in the expression of these ethylene-related genes and proteins revealed differences in the basic ability of ethylene signal transduction between CP and SC. The lower levels of the ETR and $E R F$ genes and higher levels of the $A C S$ and $A C O$ genes in CP may result in the increasing sensitivity of plants to ethylene and a higher response to ethephon stimulation in CP than in SC.

Expression offloral induction-related DEGs and DEPs responded more strongly to ethephon application in $C P$ than in $S C$

After ethephon application treatment, greater numbers of DEGs and DEPs were identified between CP and SC. In addition, the number of identified DEGs was more in SC than in CP regardless of the total or the up- and downregulated DEGs. The same trends were also observed in the DEP analysis. It seemed that SC was more responsive than $\mathrm{CP}$ to the ethephon induction. Nevertheless, the fold changes (including up and downregulation) of the DEGs and DEPs in CP, especially those involved in plant hormone signal transduction, including the $E R F$ and $A C O$ genes, were more significant than those in SC. The protein analysis revealed that the expression of ACO was higher in CP than in SC in response to ethephon induction, which was in accordance with the corresponding results of the RNA-seq analysis. These results suggested that the ethephon application treatment affected the expression of plant hormone signal transduction genes in pineapple, and stronger effects were observed in CP than in SC. Similar results were obtained for two different sensitive mandarin plants in response to ethylene application [39].

Ethephon application caused increases in carbohydrates, including sucrose and other sugars [16]. Increases in soluble sugar concentrations in the apical meristem represent one of the first physiological changes in plants and sugar and starch metabolism play vital roles in the regulation of the floral transition $[34,40]$. In the present work, the expression of the SPS, FBP and BAM genes and of some sugar metabolic proteins (GluPAT and BGLU) were upregulated and were found to be higher in CP than in SC after ethephon application. BGLU protein plays a potential role in activating glucose-conjugated compounds to migrate to shoot apical meristem for promoting flowering [41].The results above suggested that the expression of floral induction-related DEGs and DEPs responds more strongly to ethephon application in CP than in SC, which is probably a reason why CP pineapple plants could complete the floral transition in response to ethephon application and why the SC pineapple plants usually revert to vegetative growth.

DEGs associated with FMI contributed to the difference in the ethephon induction response between $C P$ and $S C$

After floral initiation in response to ethylene induction, the process of FMI and floral morphogenesis, which requires the action of several FMI and floral development genes such as FT, $L F Y, P I, C A L$ and $A G$, is initiated [12, 18, 26-27, 42-43]. TFLs prevent flowers from developing on the inflorescence apex, suppress the FMI genes $L F Y$ and $A P 1$ and maintain the inflorescence meristem [44-45]. In the present work, the expression of the pineapple $A G$ and FT genes was induced, and the expression of TFLs was repressed in response to ethephon application at 8 days after ethephon flower induction; the results were in accordance with those of previous physiological research in which, after eight days, ethephon-treated pineapple plants showed signs of inflorescence development and the phyllome stopped developing [15]. Likewise, the fold changes in the expression of the $A G$, TFL and FT genes were higher in $\mathrm{CP}$ than in SC, which probably explained why the effectiveness of ethephon application for floral induction in the pineapple plants categorized as Queen (i.e., CP) was more significant. However, these genes were not strongly induced by ethephon application, which was probably related to why pineapple plants categorized as Cayenne (i.e., SC) usually reverted to vegetative growth instead of reproductive growth. 
Liu et al.: Comparative Transcriptome and Proteome Analyses of Ethephon-Induced

Pineapple Floral Transition

More TFs responded to ethephon stimulation in SC after ethephon treatment than in CP

A higher concentration of ethephon might be perceived as one type of stress encountered by pineapple plants, which results in the increased expression of TFs after ethephon treatment [17-18]. In addition to the previously mentioned $E R F \mathrm{~s}$, other TFs, including MYBs, $W R K Y$ s, NACs and $b H L H s$, were shown to be upregulated in response to ethephon treatment. Similar results were reported in litchi [46], in which TFs were upregulated in response to ethephon during fruit abscission. These TFs are activated and subsequently regulate both the production of downstream stress defenses and the formation of protection [46-47]. Within two contrasting melon genotypes with respect to sensitivity to powdery mildew, TFs responded more strongly to pathogen attack in the resistant genotypes after Podosphaera xanthii inoculation [47]. In the present work, more TFs responded to ethephon stimulation in SC (in which relatively greater expression changes occurred) than in CP, which suggested that SC responded more strongly to defend against the ethephon stimulation stress. This phenomenon is probably why the SC plants were not strongly induced by ethephon application to transition to flowering.

In particular, MYB and WRKY TFs responded much more strongly in SC after ethephon application than in $\mathrm{CP}$, indicating greater numbers of upregulated $M Y B$ and $W R K Y$ genes and greater fold changes in the induced genes that were upregulated. Relatively high expression levels of MYB2 [48] and WRKY [49-50] TFs delay or negatively regulate flowering. Compared with wild-type Arabidopsis plants, MYB2 transgenic Arabidopsis plants displayed delayed flowering and exhibited lower expression of $C O, F T, S O C 1, L F Y$ and $A P 1$. However, different WRKY TFs such as WRKY20 [51], WRKY11.1 and WRKY11.2 [49], WRKY12 and WRKY13 [5052] have shown opposite functions in floral regulation. In this work, the MYB and WRKY TFs in SC responded much more strongly to ethephon application than did those in CP; however, additional investigations are needed to verify whether this result is related to the incompletion of the floral transition in SC.

\section{Signal transduction pathways responded differently between CP and SC}

GO annotation and KEGG analyses revealed that the ethephon-induced DEGs and DEPs were annotated predominantly to GO terms such as carbohydrate metabolism, plant hormone signal transduction and response to stimulus, as well as the KEGG pathways of starch and sucrose metabolism and plant hormone signal transduction. These results indicated that carbohydrate metabolism and plant hormone signal transduction and metabolism were commonly involved in the floral transition process induced by ethephon. Similar results have been reported previously [18]. However, more carbohydrate metabolism, plant hormone signal transduction and metabolism-associated GO terms and KEGG metabolic pathways with more DEGs were enriched in the CP-T and CP-CK comparison group than in the SC-T and SC-CK comparison group. In addition, a few photoperiodism-related GO terms, including photosynthesis, light reaction and photosynthesis, and light harvesting as well as KEGG pathways, namely, photosynthesis and circadian rhythm-plant [34], were annotated in the CP-T and CP-CK comparison group. Combined with the photoperiod pathway-associated DEGs, COR27 and GI $[42,53]$, identified in CP (not showed), these results suggested that ethephon induction probably triggered the photoperiod pathway for floral initiation in the $\mathrm{CP}$ pineapple plants, whereas the corresponding photoperiod pathway was not triggered in the SC plants.

Notably, few DEPs identified in this work were associated with plant floral transition and the FMI process. Additionally, the correlation coefficient was relatively lower in this work when the correlation between RNA and protein expression was explored. This result was probably due to the presence of posttranscriptional regulation and posttranslational modifications that occurred during the floral induction process in response to ethephon stimulation. Remarkably, the change tendency of the $A C O$ gene was in accordance with the ACO proteins in response to ethephon treatment. $A C O$ is an upstream gene in the ethephoninduced floral transition process; $A C O$ is the key regulatory gene in the triggering of flowering $[12,23,38]$.

Despite the aforementioned reasons explaining the cause of variation in response to the ethephon-induced floral transition between CP and SC, additional research is needed to verify the expression of genes and proteins in the corresponding transgenic pineapple plants. 


\section{Cellular Physiology Cell Physiol Biochem 2018;50:2139-2156 \begin{tabular}{l|l|l} 
and Biochemistry Published online: 10 November 2018 & $\begin{array}{l}\text { (c) } 2018 \text { The Author(s). Published by S. Karger AG, Basel } \\
\text { www.karger.com/cpb }\end{array}$
\end{tabular} \\ Liu et al.: Comparative Transcriptome and Proteome Analyses of Ethephon-Induced \\ Pineapple Floral Transition}

\section{Conclusion}

A basic difference exists between the two types of pineapple. The ability for ethylene signal transduction was higher in CP than in SC. After ethephon treatment, the DEGs and DEPs involved in plant hormone signal transductionand carbohydrate metabolism and FMI were more differentially expressed in CP than in SC. More TFs including MYBs, WRKYS and $N A C$ s, responded to ethephon stimulation in SC than in CP. In addition to the shared metabolic pathways such as carbohydrate metabolism and plant hormone signal transduction, ethephon induction also probably triggered the photoperiod pathway for floral initiation in the CP pineapple plants. These findings contribute to the understanding of the molecular mechanism underlying the variation in responses to ethephon-induced floral transition between CP and SC and provide a rich database for mining for the functional analysis of pineapple cultivation and molecular-assisted breeding.

\section{Acknowledgements}

This work was financially supported by the Provincial Grants (2017A020208018) of SciTechnology from Guangdong Province, P. R. China. We thank GENE DENOVO Biotechnology Co. (Guangzhou) for their assistance with the bio-information analyses.

\section{Disclosure Statement}

The authors declare that there are no conflicts of interest.

\section{References}

1 Smith LB, Downs RJ: Bromelioides (Bromeliaceae). Flora neotropica, monographno. New York, Hafner Press, 1974.

- Jiang ZD, Zheng H, Mantri N, Qi ZC, Zhang XD, Hou ZN, Chang JD, Lu HF, Liang ZS: Prediction of relationship between surface area, temperature, storage time and ascorbic acid retention of fresh-cut pineapple using adaptive neuro-fuzzy inference system (ANFIS). Postharvest Biol Tec 2016;113:1-7.

3 Chauhan OP, Shah A, Singh A, Raju PS, Bawa AS: Modeling of pre-treatment protocols for frozen pineapple slices. LWT-Food Sci Technol 2009;42:1283-1288.

4 Hossain MA, Rahman SMM: Total phenolics, flavonoids and antioxidant activity of tropical fruit pineapple. Food Res Int 2011;44:672-676.

-5 Steingass CB, Langen J, Carle R, Schmarr HG: Authentication of pineapple (Ananas comosus [L.] Merr.) fruit maturity stages by quantitative analysis of $\gamma$ - and $\delta$-lactones using headspace solid-phase microextraction and chirospecific gas chromatography-selected ion monitoring mass spectrometry (HS-SPME-GC-SIM-MS). Food Chem 2015;168:496-503.

6 Dorey E, Fournier P, Léchaudel M, Tixier P: Modeling sugar content of pineapple under agro-climatic conditions on Reunion Island. Eur J Agron 2016;73:64-72.

7 Bernier G, Havelange A, Houssa C, Petitjean A, Lejeune P: Physiological signals that induce flowering. Plant Cell 1993;5:1147-1155.

-8 Qian HF, Han X, Peng XF, Lu T, Liu WP, Fu ZW: The circadian clock gene regulatory module enantio selectively mediates imazethapyr-induced early flowering in Arabidopsis thaliana. J Plant Physiol 2014;171:92-98.

-9 Dass HC, Randhawa GS, Negi SP: Flowering in pineapple as influenced by ethephon and its combinations with urea and calcium carbonate. Sci Hortic 1975;3:231-238.

10 Dass HC, Randhawa GS, Singh HP, Ganapathy KM: Effect of pH and urea on the efficacy of ethephon for induction of flowering in pineapple. Sci Hortic 1976;5:265-268. 


\section{Cellular Physiology Cell Physiol Biochem 2018;50:2139-2156 \begin{tabular}{l|l|l} 
and Biochemistry Published online: 10 November 2018 & $\begin{array}{l}\text { (c) } 2018 \text { The Author(s). Published by S. Karger AG, Basel } \\
\text { www.karger.com/cpb }\end{array}$ \\
\hline
\end{tabular}}

Liu et al.: Comparative Transcriptome and Proteome Analyses of Ethephon-Induced

Pineapple Floral Transition

11 Bartholomew DP, Paul RE, Rohrbach KG: Crop environment, plant growth and physiology; in Bartholomew DP, Paul RE, Rohrbach KG (eds): The Pineapple: Botany, Production and Uses. Wallingford, UK, CABI Publishing, 2003, pp 69-108.

-12 Espinosa MEÁ, Moreira RO, Lima AA, Ságio SA, Barreto HG, Luiz SLP, Abreu CEA, Yanes-Paz E, Ruíz YC, González-Olmedo JL, Chalfun-Júnior A: Early histological, hormonal, and molecular changes during pineapple (Ananas comosus (L.) Merrill) artificial flowering induction. J Plant Physiol 2017;209:11-19.

13 Bartholomew DP, Malezieux E, Sanewski GM, Sinclair E: Inflorescence, and fruit development and yield; in Bartholomew DP, Paull RE, Rohrbach KG (eds): The Pineapple: Botany, Production and Uses. CABI Publishing, UK, Wallingford, 2003; pp 167-202.

14 Van de Poel B, Ceusters J, De Proft MP: Determination of pineapple (Ananas comosus, MD-2 hybrid cultivar) plant maturity, the efficiency of flowering induction agents and the use of activated carbon. Sci Hortic 2009;120:58-63.

15 Liu SH, Zang XP, Sun GM: Changes in endogenous hormone concentrations during inflorescence induction and development in pineapple (Ananas comosus cv. Smooth Cayenne) by ethephon. Afri J Biotechnol 2011;10:10892-10899.

16 Ávila M, Blanco MA, Nieves N, González J, Marrero P, González A, Quiñones J, Martínez T: Effect of ethrel on flowering induction in pineapple (Ananas comosus (L.) Merr.) smooth cayenne cv. Serrana. i. changes in levels of polyamines, proteins and carbohydrates. Acta Horticul 2005;666:175-182.

17 Li YH, Wu QS, Huang X, Liu SH, Zhang HN, Zhang Z, Sun GM: Molecular cloning and characterization of four genes encoding ethylene receptors associated with pineapple (Ananas comosus L.) flowering. Front Plant Sci 2016;7:710.

-18 Liu CH, Fan C: De novo transcriptome assembly of floral buds of pineapple and identification of differentially expressed genes in response to ethephon induction. Front Plant Sci 2016;7:203.

19 Yang SF, Hoffman NE: Ethylene biosynthesis and its regulation in higher plants. Annu Rev Plant Physiol 1984;35:155-189.

20 Kende H: Ethylene biosynthesis. Annu Rev Plant Physiol Plant Mol Biol 1993;44:283-307.

-21 Cazzonelli CI, Cavallaro AS, Botella JR: Cloning and characterization of ripening-induced ethylene biosynthetic genes from non -climacteric pineapple (Ananas comosus) fruits. Aust J Plant Physiol 1998;25:513-518.

22 Botella JR, Cavallaro AS, Cazzonelli CI: Towards the production of transgenic pineapple to control flowering and ripening. Acta Horticul 2000;529:115-122.

23 Trusov Y, Botella JR: Silencing of the ACC synthase gene ACACS2 causes delayed flowering in pineapple [Ananas comosus (L.) Merr.]. J Exp Bot 2006;57:3953-3960.

24 Wang RH, Hsu YM. Bartholomew DP, Maruthasalam S, Lin CH: Delaying natural flowering in pineapple through foliar application of Aviglycine, an inhibitor of ethylene biosynthesis. Hortscience 2007;42:11881191.

25 Chang JC, Maruthasalam S, Liu YL, Sun CM, Shih WS, Lee PF, Lin CH: Forcing of 'Tainon17' pineapple with calcium carbide $\left(\mathrm{CaC}_{2}\right)$ and/or ice-cold stress under field conditions. Acta Horticul 2011;902:327-335.

-26 Lv LL, Duan J, Xie JH, Liu YG, Wei CB, Liu SH, Zhang JX, Sun GM: Cloning and expression analysis of a PISTILLATA homologous gene from pineapple (Ananas comosus L. Merr). Int J Mol Sci 2012;13:1039-1053.

-27 Lv LL, Duan J, Xie JH, Wei CB, Liu YG, Liu SH, Sun GM: Isolation and characterization of a FLOWERING LOCUS $T$ homolog from pineapple (Ananas comosus (L.) Merr). Gene 2012;505:368-373.

28 Turnbull CGN, Sinclair ER, Anderson KL, Nissen RJ, Shorter AJ, Lanham TE: Routes of ethephon uptake in pineapple (Ananas comosus) and reasons for failure of flower induction. J Plant Growth Reg 1999;18:145152.

29 De Greef JA, De Proft MP, Mekers O, Van Dijck R, Jacobs L, Philippe L: Floral induction of bromeliads by ethylene; in Clijsters H, De Proft M, Marcelle R, Van Poucke M (eds): Biochemical and physiological aspects of ethylene production in lower and higher plants. Advances in Agricultural Biotechnology, Dordrecht, Springer, 1989, pp 313-322.

30 Lin CH, Maruthasalam S, Shiu LY, Lien WC, Loganathan M, Yu CW, Hung SH, Ko Y, Chen YY: Physical and chemical manipulation of flowering in pineapple. Acta Horticul 2009;822:117-124.

-31 Ming R, VanBuren R, Wai CM, Tang HB, Schatz MC, Bowers JE, Lyons E, Wang ML, Chen J, Biggers E, Zhang J, Huang L, Zhang L, Miao W, Zhang J, Ye Z, Miao C, Lin Z, Wang H, Zhou H et al.: The pineapple genome and the evolution of CAM photosynthesis. Nat Genet 2015;47:1435-1445. 


\section{Cellular Physiology Cell Physiol Biochem 2018;50:2139-2156 \begin{tabular}{l|l|l|l}
\hline and Biochemistry $10.1159 / 000495057$ & (c) 2018 The Author(s). Published by S. Karger AG, Basel \\
Published
\end{tabular}

Liu et al.: Comparative Transcriptome and Proteome Analyses of Ethephon-Induced

Pineapple Floral Transition

-32 Kanehisa M, Araki M, Goto S, Hattori M, Hirakawa M, Itoh M, Katayama T, Kawashima S, Okuda S, Tokimatsu T, Yamanishi Y: KEGG for linking genomes to life and the environment. Nucleic Acids Res 2008;36:480-484.

33 Ashburner M, Ball CA, Blake JA, Botstein D, Butler H, Cherry JM, Davis AP, Dolinski K, Dwight SS, Eppig JT, Harris MA, Hill DP, Issel-Tarver L, Kasarskis A, Lewis S, Matese JC, Richardson JE, Ringwald M, Rubin GM, Sherlock G: Gene Ontology: tool for the unification of biology. Nat Genet 2000;25:25-29.

-34 Guo XL, Yu C, Luo L, Wan HH, Li YS, Wang J, Cheng TR, Pan HT, Zhang QX: Comparative transcriptome analysis of the foral transition in Rosa chinensis 'Old Blush' and R. odorata var. gigantean. Sci Rep 2017;7:6068.

35 Zhou Q, Guo JJ, He CT, Shen C, Huang YY, Chen JX, Guo JH, Yuan JG, Yang ZY: Comparative transcriptome analysis between low- and high-cadmium-accumulating genotypes of pakchoi (Brassica chinensis L.) in response to cadmium stress. Environ Sci Technol 2016;50:6485-6494.

-36 Wuriyanghan H, Zhang B, Cao WH, Ma B, Lei G, Liu YF, Wei W, Wu, HJ, Chen LJ, Chen HW, Cao YR, He SJ, Zhang WK, Wang XJ, Chen SY, Zhang JS: The ethylene receptor ETR2 delays floral transition and affects starch accumulations in rice. Plant Cell 2009;21:1473-1494.

37 Wang F, Cui X, Sun Y, Dong CH: Ethylene signaling and regulation in plant growth and stress responses. Plant Cell Rep 2013;32:1099-1109.

38 Choudhury SR, Roy S, Sengupta DN: Characterization of transcriptional profiles of MA-ACS1 and MA-ACO1 genes in response to ethylene, auxin, wounding, cold and different photo periods during ripening in banana fruit. J Plant Physiol 2008;165:1865-1878.

-39 Alós E, Distefano G, Rodrigo MJ, Gentile A, Zacaŕas L: Altered sensitivity to ethylene in 'Tardivo', a lateripening mutant of Clementine mandarin. Physiol Plantarum 2014;151:507-521.

40 Matsoukas IG: Interplay between sugar and hormone signaling pathways modulate floral signal transduction. Front Genet 2014;5:218-218.

-41 Coneva V, Zhu T, Colasanti J: Expression differences between normal and indeterminate1 maize suggest downstream targets of ID1, a floral transition regulator in maize. J Exp Bot 2007;58:3679-3693.

42 Wang P, Cui X, Zhao C, Shi L, Zhang G, Sun F, Cao X, Yuan L, Xie Q, Xu X: COR27 and COR28 encode nighttime repressors integrating Arabidopsis circadian clock and cold response. J Integr Plant Biol 2017;59:78-85.

43 Wang JB, Li ZY, Lei M, Fu YL, Zhao JJ, Ao MF, Xu L: Integrated DNA methylome and transcriptome analysis reveals the ethylene-induced flowering pathway genes in pineapple. Sci Rep 2018;7:17167.

44 Alvarez J, Guli CL, Yu XH, Smyth DR: terminal flower: a gene affecting inflorescence development in Arabidopsis thaliana. Plant J 1992;2:103-116.

45 Kotoda N, Wada M: MdTFL1, a TFL1-like gene of apple, retards the transition from the vegetative to reproductive phase in transgenic Arabidopsis. Plant Sci 2005;168:95-104.

46 Li CQ, Wang Y, Ying PY, Ma WQ Li JG: Genome-wide digital transcript analysis of putative fruitlet abscission related genes regulated by ethephon in litchi. Front Plant Sci 2015;6:502.

47 Zhu QL, Gao P, Wan Y, Cui HN, Fan C, Liu S, Luan FS: Comparative transcriptome profiling of genes and pathways related to resistance against powdery mildew in two contrasting melon genotypes. Sci Hortic 2018;227:169-180.

-48 Shan H, Chen SM, Jiang JF, Chen FD, Chen Y, Gu CS, Li PL, Song AP, Zhu XR, Gao HS, Zhou GQ, Li T, Yang $\mathrm{X}$ : Heterologous expression of the chrysanthemum R2R3-MYB transcription factor CmMYB2 enhances drought and salinity tolerance, increases hypersensitivity to ABA and delays flowering in Arabidopsis thaliana. Mol Biotechnol 2012;51:160-173.

49 Cai YH, Chen XJ, Xie K, Xing QK, Wu YW, Li J, Du CH, Sun ZX, Guo ZJ: Dlf1, a WRKY transcription factor, is involved in the control of flowering time and plant height in rice. PLoS One 2014;9:102529.

50 Li W, Wang HP, Yu DQ: Arabidopsis WRKY transcription factors WRKY12 and WRKY13 oppositely regulate flowering under short-day conditions. Mol Plant 2016;9:1492-1503.

51 Luo X, Sun XL, Liu BH, Zhu D, Bai X, Cai H, Ji W, Cao L, Wu J, Wang MC, Ding XD, Zhu YM: Ectopic expression of a WRKY homolog from Glycine soja alters flowering time in Arabidopsis. PLoS One 2013;8:e73295.

-52 Yu YC, Hu RB, Wang HM, Cao YP, He G, Fu CX, Zhou GK: MIWRKY12, a novel Miscanthus transcription factor, participates in pith secondary cell wall formation and promotes flowering. Plant Sci 2013;212:1-9.

53 Ding JH, Böhlenius H, Rühl MG, Chen P, Sane S, Zambrano JA, Zheng B, Eriksson ME, Nilsson O: GIGANTEAlike genes control seasonal growth cessation in Populus. New Phytol 2018;218:1491-1503. 